\title{
Bilateral Anterior Ischemic Optic Neuropathy During Treatment with Pegylated Interferon and Ribavirin
}

\author{
Jason Rinehart OD FAA0; Wilmington VA Medical Center, Wilmington, Delaware \\ Lisa Stottlemyer OD FAA0; Wilmington VA Medical Center, Wilmington, Delaware \\ Correspondence may be directed to jason.rinehart@va.gov
}

\begin{abstract}
Hepatitis C virus (HCV) is a blood-borne pathogen that often results in chronic liver infection leaving patients at risk for cirrhosis, liver decompensation and hepatocellular carcinoma. The goal of therapy is to eliminate the virus in order to minimize morbidity and mortality from HCV infection. Traditional treatment has utilized a combination of pegylated-interferon (IFN) and ribavirin that often causes both systemic and ocular side effects. The most common ocular side effects are retinal hemorrhages and cotton wool spots, although in rare circumstances more significant ophthalmic adverse events have been attributed to IFN therapy.

Here we discuss a case of bilateral anterior ischemic optic neuropathy (AION) that occurred during treatment with IFN and ribavirin for HCV genotype 1. We review the proposed pathogenesis of ocular side effects associated with this regimen, as well as the pathogenesis and risk factors associated with AION itself. Finally, we will offer clinical recommendations for screening for more than retinopathy should a patient present with ophthalmic complications.
\end{abstract}

Keywords: interferon, hepatitis C, anterior ischemic optic neuropathy, retinopathy, side effects

\section{Introduction}

Hepatitis $\mathrm{C}$ virus (HCV) is a blood-borne pathogen classified into 6 major genotypes that can cause both acute and chronic liver infection. While $15-25 \%$ of those infected may clear the virus without treatment, the majority of individuals will develop chronic liver infection leaving them at risk for cirrhosis, liver decompensation, and hepatocellular carcinoma. ${ }^{1}$ It is estimated that chronic HCV infection affects between 130-150 million people worldwide. ${ }^{1,2}$

The goal of therapy is to eliminate the virus in order to minimize morbidity and mortality from HCV infection. Until recently, the mainstay of therapy has been a combination of pegylated-interferon alpha (IFN) and ribavirin over a course of 48 weeks. Side effects of this regimen are essentially inevitable and can be quite severe. Rapid advances in HCV treatments offer higher cure rates and fewer side effects than IFN therapy. However, these emerging therapies are not yet widely employed in the treatment of $\mathrm{HCV}$, mainly due to the significant cost burden associated with these regimens. 3

The ophthalmic side effects of IFN therapy are well established, most commonly involving retinal hemorrhage and cotton wool spots, which often go unnoticed due to the lack of associated visual disturbance. In rare circumstances, more significant ophthalmic adverse events, including central retinal vein occlusion (CRVO) and anterior ischemic optic neuropathy (AION), have been attributed to IFN therapy. As these more ominous ocular sequelae require discontinuation of treatment to allow for visual improvement, it would behoove the clinical optometrist to be able to recognize these adverse events and their association with IFN use.

\section{Case Report}

A 55-year-old white male presented to the eye clinic reporting a visual disturbance described as "missing spots" in his vision for one week. The patient's ocular history was unremarkable. His systemic history was notable for hypertension controlled with hydrochlorothiazide/lisinopril $20 \mathrm{mg}$ daily and chronic hepatitis $\mathrm{C}$ genotype 1 treated with ribavirin $1200 \mathrm{mg}$ daily and pegylated-inferferon alpha-2A injections $180 \mu \mathrm{g}$ weekly. The patient had tolerated this therapy well and had no detectable virus by his $20^{\text {th }}$ week of treatment. By his $22^{\text {nd }}$ week he developed ribavirin-induced anemia with symptoms 
including shortness of breath, dizziness and fatigue. These symptoms improved with a reduction in dose from $1200 \mathrm{mg}$ to $800 \mathrm{mg}$ daily. Four weeks prior to his presentation to the eye clinic, the patient had developed an intermittent fever with myalgia, nausea, vomiting and diarrhea. At that time, he was evaluated in the emergency room with a fever of $103.7^{\circ} \mathrm{F}$ and reduced blood pressure measuring 99/66.

At presentation to the eye clinic, the patient was in his $44^{\text {th }}$ week of interferon therapy. His visual acuities were 20/30 (6/9) in the right eye (OD) and 20/25 (6/7.5) in the left eye (OS). The pupils were round and equally reactive to light, without an afferent pupillary defect. Confrontation visual fields were restricted superiorly in each eye. Slit lamp examination was remarkable for trace nuclear sclerotic cataracts bilaterally. Goldmann applanation tonometry readings were $9 \mathrm{~mm}$ $\mathrm{Hg}$ OD and $9 \mathrm{~mm} \mathrm{Hg}$ OS at 9:15am. Blood pressure was measured at 112/65 RAS. Dilated fundus exam revealed subtle, sectoral optic disc edema associated with flame-shaped hemorrhages. In addition, focal retinal arteriole occlusions were present bilaterally (Figure 1). Automated visual field testing demonstrated a superior altitudinal defect in each eye corresponding to the areas of greatest optic disc edema.

Given the atypical presence of bilateral optic nerve head edema, a MRI was obtained which did not reveal any intracranial abnormalities or enhancement of the optic nerves. Given the severity of his systemic symptoms, he was admitted and underwent an infectious disease workup. The patient was ultimately diagnosed with interferon-associated retinopathy in conjunction with bilateral non-arteritic anterior ischemic optic neuropathy. The findings were relayed to the patient's gastroenterologist with the recommendation to discontinue interferon and ribavirin treatment.

The patient was monitored in the eye clinic over a 6-week period, during which time his symptoms subsided, his visual acuity returned to 20/20 (6/6) in each eye, and his optic disc edema and retinal findings gradually resolved (Figures 2 and 3). Retinal arteriolar occlusions and anterior ischemic optic neuropathy were both considered as contributory to visual field loss at presentation. Insofar as the improvement of disc edema yielded improvement in the visual field defect, the visual field defect was most likely a manifestation of AION.

Treatment with interferon and ribavirin had yielded an end of treatment response with no virus detectable by the $20^{\text {th }}$ week, remaining so until treatment was discontinued in his $44^{\text {th }}$ week. After treatment was discontinued, the patient relapsed and virus was detectable several months later. Treatment was eventually restarted with ledipasvir 90mg/sofosbuvir 400mg (Harvoni) and ribavirin $1200 \mathrm{mg}$, both daily. He has had no detectable virus at weeks 4,8 and 12 post-re-initiation of treatment.

\section{Discussion}

Interferons are naturally occurring glycoproteins with anti-viral, anti-tumor and anti-angiogenic properties. ${ }^{4,5}$ For the treatment of HCV, IFN is usually administered weekly by subcutaneous injection. Patients on this regimen commonly experience fever, chills and flu-like symptoms; nausea, vomiting and systemic hypotension can occur, albeit rarely. ${ }^{4,6}$ Reports of incidence of retinopathy vary widely, attributed to differences in study design. Lack of formal screening leads to under-reporting, while evaluation of symptomatic or at-risk patients leads to over-reporting of ocular sequelae. ${ }^{7}$ Raza et al report the overall incidence of retinopathy in patients treated with IFN and ribavirin to be approximately $27 \%$; patients with diabetes (DM) and hypertension (HTN) are 5 to 6 times more likely to develop retinopathy than patients without these systemic conditions. ${ }^{5}$

Interferon-associated retinopathy typically occurs 2 to 12 weeks after initiation of therapy. ${ }^{7}$ Patients are generally visually asymptomatic, but exhibit retinal hemorrhages and/or cotton wool spots on dilated fundus examination. These changes are proposed to be the end result of disturbances in retinal microvascular circulation. ${ }^{8}$ The pathophysiology is not fully understood, but many suggest a role of endothelial dysfunction where platelet aggregation with leukocyte adherence to the vascular endothelium form microthrombi capable of focal microinfarction. ${ }^{7,-12}$

Interferons act by binding to receptor cells and initiating the production of effector proteins. In the event of an interferonassociated AION, it is theorized that these circulating proteins could lead to hyperviscosity and result in compromised perfusion to the capillary beds of the optic nerve head. ${ }^{6}$ Hayreh has long argued that AION results from a transient decrease in perfusion of the optic nerve head, usually during sleep, and is therefore a hypotensive event rather than an embolic disorder. ${ }^{13-16}$ Systemic hypotension is a well-known finding associated with IFN treatment. Our patient was hospitalized with a hypotensive event in the weeks preceding the onset of visual symptoms.

No standard recommendations for the screening of patients for interferon-associated retinopathy exist. This is likely due to the fact that the retinopathy is generally not associated with vision loss, follows a benign course, and is self-limiting, usually resolving shortly after the completion of the course of treatment. In general, most clinicians agree that screening high risk patients for interferon-retinopathy is prudent, however, O'Day et al suggest that routine screening is unnecessary, even for patients with HTN and DM, unless they are visually symptomatic. $^{7}$ As vision loss in patients with identified IFNassociated retinopathy is rare, treatment is generally continued despite retinopathy being identified especially given the greater risk to the overall health of a patient with premature cessation 
of interferon treatment. However, if vision loss occurs during treatment, suspicion for a less common adverse event such as
CRVO or AION should be explored. In such cases, the best visual outcome exists with cessation of treatment. ${ }^{12}$

Figure 1

DAY 1
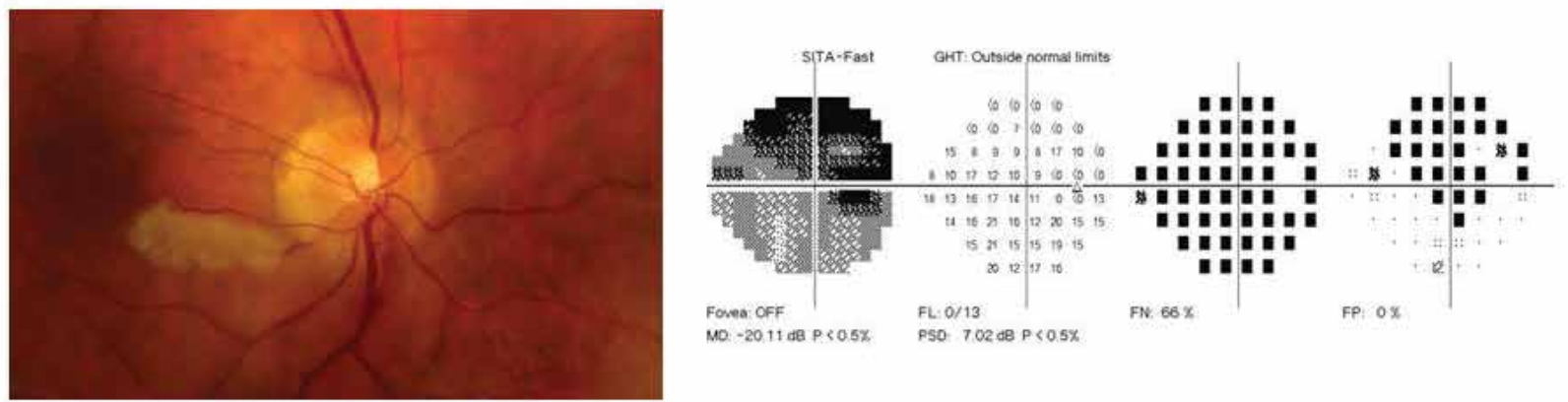

Fovea: OFF

MO -2011 de $P<0.5 x$

FL: $0 / 13$

FN. $66 x$
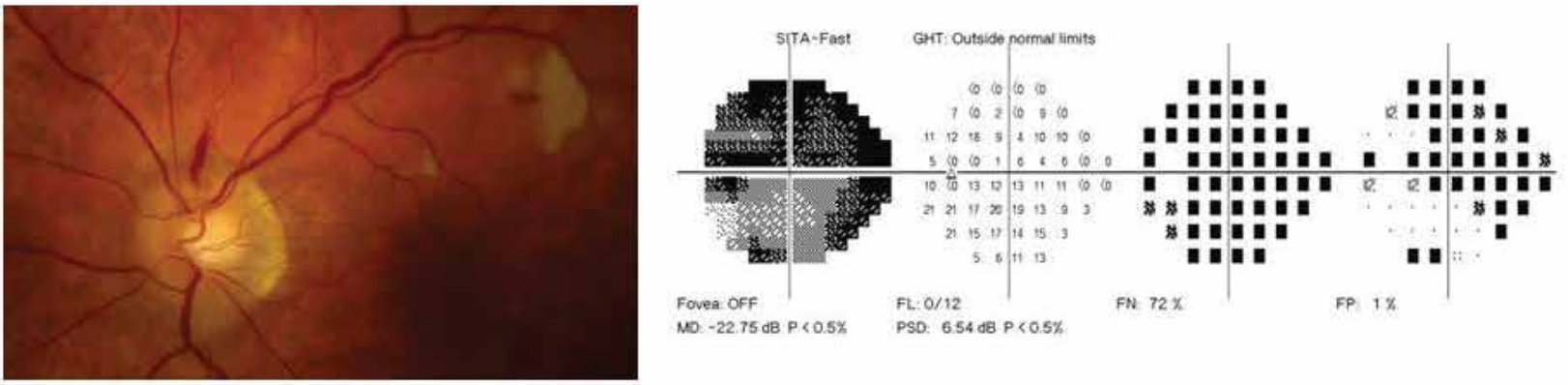

Fover OFF

MO -22.75 dB $P<0.5 \%$

FL: $0 / 12$

PSD. $6.54 \mathrm{~d} 8 \mathrm{P}<0.5 \%$

FN. $72 x$

FP $1 \%$

10

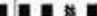

10 10

1.

Figure 2

\section{DAY 12}
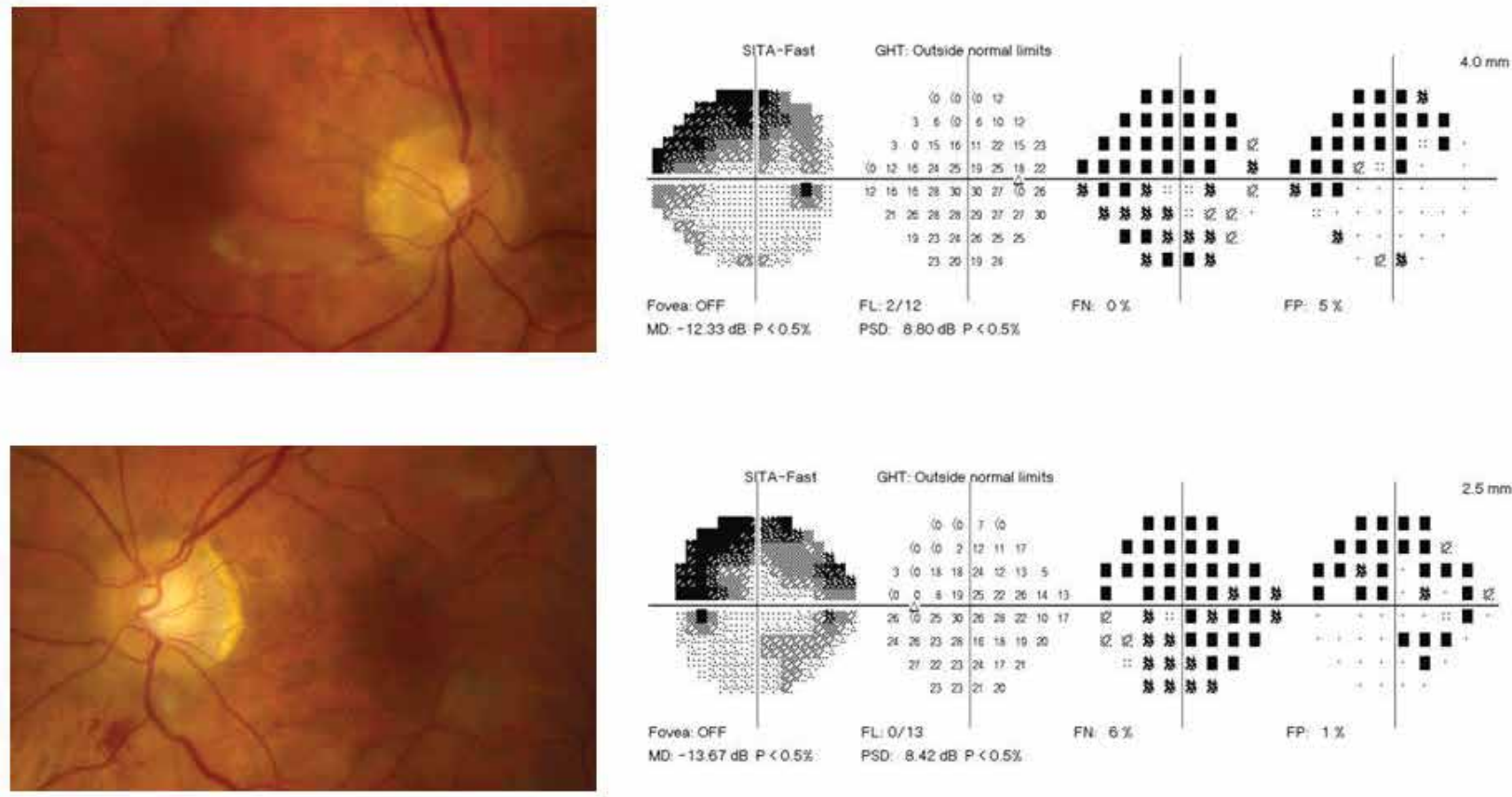
Figure 2

\section{DAY 45}
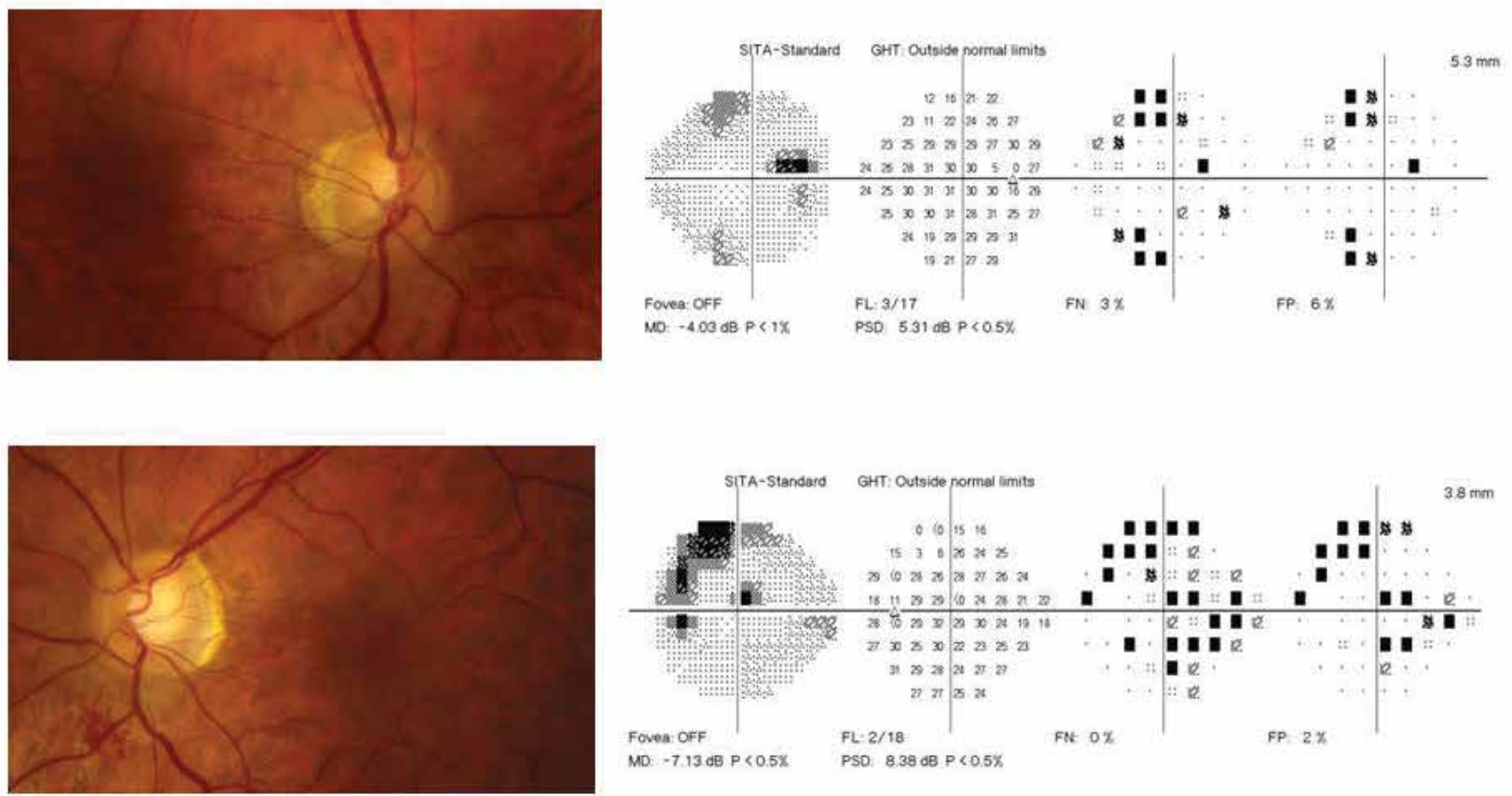

\section{Final Thoughts}

In the ever-changing landscape of HCV treatment, pegylatedinterferon seems likely to have a reduced presence in many treatment protocols, as direct-acting antiviral agents such as Sovaldi and Harvoni offer shorter treatment courses, superior cure rates, and fewer side effects. However, for a number of reasons, including treatment cost, IFN will continue to have a place in the armamentarium of HCV treatment, and an understanding of potential ocular sequelae associated with this regimen remains necessary to allow for optimal care of these patients.

\section{References}

1. Centers for Disease Control and Prevention. Viral Hepatitis - Hepatitis C Information [Internet]. Atlanta, GA: Centers for Disease Control and Prevention; [updated 2015 May 31; cited 2015 July 9]. Available from: http://www.cdc.gov/hepatitis/hcv/hcvfaq.htm\#section 1

2. World Health Organization. Media Centre Hepatitis C Fact Sheet [Internet]. Geneva Switzerland: World Health Organization; [updated 2014 April; cited 2015 July 9]. Available from: http://www.who.int/ mediacentre/factsheets/fs164/en/

3. Kohli A, Shaffer A, Sherman A, et al. Treatment of hepatitis C: a systematic review. JAMA. 2014 Aug 13;312(6):631-40.

4. Fraunfelder FW, Fraunfelder FT. Interferon alfa-associated anterior ischemic optic neuropathy. Ophthalmology. 2011 Feb;118(2):408-11.e1-2.
5. Raza A, Mittal S, Sood GK. Interferon-associated retinopathy during the treatment of chronic hepatitis C: a systematic review. J Viral Hepat. 2013 Sep;20(9):593-9.

6. Purvin VA. Anterior ischemic optic neuropathy secondary to interferon alfa. Arch Ophthalmol. 1995 Aug;113(8):1041-4.

7. O’Day R, Gillies MC, Ahlenstiel G. Ophthalmologic complications of antiviral therapy in hepatitis C treatment. World J Gastroenterol. 2013 Dec 7;19(45):8227-37.

8. Okuse C, Yotsuyanagi H, Nagase Y, et al. Risk factors for retinopathy associated with interferon alpha- $2 \mathrm{~b}$ and ribavirin combination therapy in patients with chronic hepatitis C. World J Gastroenterol. 2006 Jun 21;12(23):3756-9.

9. Nagaoka T, Sato E, Takahashi A, et al. Retinal circulatory changes associated with interferon-induced retinopathy in patients with hepatitis C. Invest Ophthalmol Vis Sci. 2007 Jan;48(1):368-75.

10. Guyer DR, Tiedeman J, Yannuzzi LA, et al. Interferon-associated retinopathy. Arch Ophthalmol. 1993 Mar;111(3):350-6.

11. Nishiwaki H, Ogura Y, Miyamoto K, et al. Interferon alfa induces leukocyte capillary trapping in rat retinal microcirculation. Arch Ophthalmol. 1996 Jun;114(6):726-30.

12. Selcukbiricik F, Tural D, Senel TE, et al. Bilateral Ischemic Optic Neuropathy Developed under Interferon Therapy. Case Rep Ophthalmol Med. 2012;2012:102739.

13. Hayreh SS. Ischemic optic neuropathies - where are we now? Graefes Arch Clin Exp Ophthalmol. 2013 Aug;251(8):1873-84.

14. Miller NR. Current concepts in the diagnosis, pathogenesis, and management of nonarteritic anterior ischemic optic neuropathy. J Neuroophthalmol. 2011 Jun;31(2):e1-3. 